Sitzungsberichte

der Akademie der Wissenschaften

der DDR

Jahrgang $1980 \cdot$ Nr. 15/N

Mathematik - Naturwissenschaften - Technik

\title{
Umwelttoxikologische Bewertung
} chemischer Produkte

AKADEMIE-VERLAG BERLIN 1981

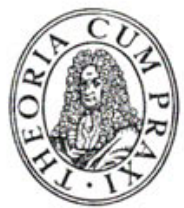


Vorträge gehalten in der Sitzung der

Klasse Medizin am 21.9. 1978 und in der

gemeinsamen Sitzung der Klassen Medizin und Umweltschutz

und Umweltgestaltung am 22.11. 1979

Herausgegeben im Auftrage

des Präsidenten der Akademie

der Wissenschaften der DDR

von Vizepräsident Prof. Dr. Heinrich Scheel

Erschienen im Akademie-Verlag, DDR-1080 Berlin, Leipziger Str. 3-4

(c) Akademie-Verlag,Berlin 1980

Lizenznummer: $202: 100 / 84 / 80$

Gesamtherstellung: VEB Druckhaus Köthen

Bestellnummer: $7628882(2010 / 80 / 15 / \mathrm{N})$ LSV 2065

Printed in GDR

DDR $6,50 \mathrm{M}$ 


\section{Inhalt}

KanLhersz Lohs, Ordentliches Mitglied der Akademie der Wissenschaften der DDR Umwelttoxikologische Bewertung chemischer Erzeugnisse und ihrer Abprodukte ..

Helaut Haener, Ordentliches Mitglied der Akademie der Wissenschaften der DDR

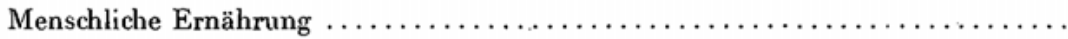

Rudolf Exgst, Korrespondierendes Mitglied der Akademie der Wissenschaften der DDR

Schadstoffe in der menschlichen Ernährung (Umwelt) und ihre toxikologische Be-

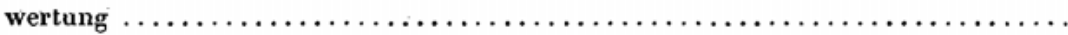

Emanuel Heinisch, Prof. Dr. sc. nat., Institut für Geographie und Geoökologie der Akademie der Wissenschaften der DDR

Okologisch-ökonomische Wechselwirkungen bei der Anwendung von Pflanzenschutzmitteln und Mitteln zur Steuerung biologischer Prozesse ................. 
